\title{
Patrones de uso de servicios entre adultos con problemas de salud mental, en Chile
}

\author{
Benjamín Vicente, ${ }^{1}$ Robert Kohn, ${ }^{2}$ Sandra Saldivia, ${ }^{1}$ \\ Pedro Rioseco ${ }^{1}$ y Silverio Torres ${ }^{1}$
}

Forma de citar Vicente B, Kohn R, Saldivia S, Rioseco P, Torres S. Patrones de uso de servicios entre adultos con problemas de salud mental, en Chile. Rev Panam Salud Publica. 2005;18(4/5):263-70.

RESUMEN Objetivo. Describir el patrón de uso de servicios generales y especializados entre los adultos con problemas de salud mental, así como su nivel de satisfacción con dichos servicios, con la finalidad de contribuir a optimizar los escasos recursos disponibles en la Región para la atención de la salud mental, particularmente en lo que corresponde a Chile.

Método. Los diagnósticos y los patrones de uso de los servicios de salud mental se obtuvieron del Estudio Chileno de Prevalencia Psiquiátrica (ECPP), una investigación representativa de la población adulta del país, basada en una muestra aleatoria estratificada de 2987 personas de 15 años y más, que abarcó el período de 1992 a 1999. Los diagnósticos psiquiátricos se obtuvieron por medio del instrumento de entrevista denominado Composite International Diagnostic Interview (CIDI). Asimismo, se preguntó a los entrevistados acerca del uso de servicios generales y de salud mental en los seis meses precedentes y sobre las dificultades que encontraron para acceder a ellos.

Resultados. Más de $44 \%$ de los entrevistados tuvieron contacto con algún tipo de servicio de salud durante los 6 meses previos al estudio, pero solo 5,6\% recibieron atención especializada. Quienes presentan los diagnósticos de trastorno obsesivo y de pánico consultan con mayor frecuencia, pero no en centros especializados. El consumo de sustancias tóxicas y la personalidad antisocial se relacionaron con un bajo nivel de consulta. El sistema formal de salud es la opción que más se menciona como recurso de ayuda. Más de 75\% refieren satisfacción o gran satisfacción con la atención recibida.

Conclusiones. Se confirma la existencia de una amplia brecha entre las necesidades de atención y el tratamiento realmente recibido. Los recursos alternativos informales y folclóricos (sacerdote, familiares, curanderos, hierbateros [yerberos], etc.) se utilizan con una frecuencia menor de la esperada. Las barreras indirectas de acceso a los servicios resultan claramente más frecuentes y se vinculan con desconocimiento y estigma. Un número importante de personas sin diagnóstico positivo en la entrevista CIDI hace uso de los servicios de salud mental.

Palabras clave Servicios comunitarios de salud mental, utilización, trastornos mentales, satisfacción del paciente.

Departamento de Psiquiatría y Salud Mental, Facultad de Medicina, Universidad de Concepción, Chile. Toda correspondencia deberá dirigirse a: Prof. B. Vicente, Casilla 160-C Concepción Chile. Teléfono: 56-41-312799. Correo electrónico: bvicent@ udec.cl

2 Department of Psychiatry and Human Behavior, Brown University, Rhode Island, Estados Unidos.
En Chile se han realizado estudios sobre la utilización de servicios y los niveles de satisfacción con la atención recibida, en poblaciones con diagnósticos psiquiátricos que consultan tanto al nivel primario de atención (1) como a servicios especializados (2). Las objeciones que se hacen a los estudios extranjeros, especialmente los norteamericanos (3-6), cuyas conclusiones sobre necesidades, costos y niveles de satisfacción pretendían extrapolarse a otros niveles 
de atención y a la comunidad en general $(7,8)$, son también aplicables a los estudios chilenos, por lo que su comparación, y más aun la extrapolación de sus resultados a la población en general, resultan difíciles y poco rigurosas.

Por esas razones y por la necesidad de contar con información actualizada que facilite una adecuada planificación e instauración de los servicios, especialmente los de salud mental, en 1994 se inició una serie de estudios encaminados a conocer y examinar las características más relevantes del uso que la población adulta de Chile hace de los servicios de salud que se le ofrecen $(9,10)$.

En Chile, la atención a la salud se provee principalmente por medio de un sistema de cobertura nacional, establecido en 1952, al cual complementa un activo sector privado que atiende a aproximadamente un tercio de la población. Al comienzo, la atención a la salud mental consistió principalmente en el tratamiento hospitalario de los casos graves. Iniciativas más recientes han reorientado los esfuerzos hacia la asistencia ambulatoria, con una mayor utilización del hospital general en sus servicios de psiquiatría de agudos, y una participación creciente del nivel primario en la pesquisa y tratamiento de enfermedades psiquiátricas. Desde comienzos del decenio pasado se avanza en la instauración de un Plan Nacional de Salud Mental y Psiquiatría, coordinado por la Unidad de mayor Salud Mental del Ministerio de Salud, cuya responsabilidad principal es la supervisión técnica y la consecución de mayor financiamiento para el sector.

Se han establecido servicios de internación y ambulatorios en los 28 servicios de salud en los que está dividido el país. Las distintas zonas geográficas están a cargo de unidades de atención primaria que prestan servicio a la población tanto urbana como rural. En una buena proporción de ellas, el personal que atiende a personas con problemas de salud mental está integrado por médico general, psicólogo, asistente social y enfermera. Esta acción se ha visto fortalecida recientemente por programas nacionales que reciben financiamiento central, como el Programa Nacional de Depresión, el cual permite atender casos leves y moderados y remitir oportunamente al nivel secundario las depresiones graves $\mathrm{y}$ otros cuadros complejos. La distribución de los recursos, en el ámbito nacional, es aún bastante desigual.

El presente trabajo se basa en los resultados de un estudio de utilización de servicios y nivel de satisfacción con la atención recibida, que forma parte del Estudio Chileno de Prevalencia Psiquiátrica (ECPP) (11), el cual se realizó en Chile entre 1992 y 1999 con la finalidad de evaluar la prevalencia y los factores de riesgo de trastornos psiquiátricos en una muestra de adultos representativa de la población nacional.

\section{MÉTODOS}

\section{Selección de la muestra}

El ECPP se basó en una muestra aleatoria que se estratificó en dos etapas. En la primera se consideraron primeramente viviendas y en la segunda, personas de 15 años y más. El estudio se diseñó de tal manera que fuera representativo de la población adulta del país. Se eligieron cuatro provincias: Santiago, Concepción, Iquique y Cautín, cada una perteneciente a una región diferente del país. Si bien la muestra se considera representativa de la población nacional, la distribución de los recursos sanitarios disponibles dista mucho de ser homogénea.

En Chile, las provincias están divididas en comunas y subsecuentemente en distritos, conformados por manzanas. Se hizo una selección aleatoria en cada uno de estos niveles hasta llegar al nivel de las viviendas. El número de viviendas que debían seleccionarse en cada manzana se calculó a partir de los datos del Censo Poblacional Nacional de 1992. Para elegir a la persona que debía entrevistarse en cada vivienda, se generó una lista de sus moradores, empezando por los varones y en orden descendente de edad, hasta un mínimo de 15 años. Luego, mediante asignación aleatoria de las 12 tablas de Kish, se seleccionó a la persona que sería entrevistada (12).

El estudio, llevado a cabo por el Departamento de Psiquiatría y Salud
Mental de la Universidad de Concepción, abarcó el período de julio de 1992 a junio de 1999. Conforme al financiamiento disponible, la investigación se realizó en etapas, que de acuerdo con las provincias estudiadas se completaron en el orden siguiente: Concepción, Santiago, Iquique y Cautín.

La tasa de respuesta fue de 90,3\%, entre un total de participantes que ascendió a 2987 al final del estudio. Varió según las provincias $\left(\chi^{2}=11,08\right.$; grados de libertad $(\mathrm{gl})=3 ; P<0,02)$. Santiago mostró la tasa más alta de falta de respuesta $(12,6 \%)$ e Iquique la más baja $(7,5 \%)$.

Se estableció un peso estadístico apropiado para calcular la probabilidad de ser seleccionado y participar en el estudio, tanto para la comuna, distrito, manzana y vivienda, como para el entrevistado. Se aplicó un segundo peso para ajustar la información al censo de 1992, a partir de la edad, el sexo y el estado civil.

\section{Diagnóstico}

El diagnóstico psiquiátrico se estableció en los términos de la versión III, revisada, del Diagnostic and Statistical Manual of Mental Disorders (DSM-III-R) (13), y se fundamentó en los datos generados por las versiones 1,0 y 1,1 de la entrevista diagnóstica internacional compuesta (Composite International Diagnostic Interview, CIDI). La CIDI está estructurada de tal manera que puedan aplicarla entrevistadores capacitados, sin necesidad de experiencia clínica (14). Al formulario de esta entrevista se agregaron dos secciones del Instrumento de Entrevista Diagnóstica (Diagnostic Interview Schedule, DIS), con el fin de cubrir el estrés postraumático y la personalidad antisocial, ya que la versión del CIDI que se utilizó no incorporaba estos trastornos (15). El DIS es un instrumento semejante en estructura y administración al CIDI, en el cual está basado. Puesto que las secciones del DIS no se administraron en Cautín, se efectuaron los ajustes y ponderaciones pertinentes para una correcta extrapolación de los resultados. La entrevista incorporó también una sección sobre la utilización de servicios. 
Los instrumentos fueron traducidos al español por el equipo investigador y validados por medio del protocolo diseñado por la Organización Mundial de la Salud (OMS). Los resultados se publicaron en su oportunidad $(16,17)$.

El Estudio Chileno de Prevalencia Psiquiátrica incluyó los siguientes diagnósticos del DSM-III-R: depresión mayor, trastorno bipolar, distimia, trastornos de ansiedad generalizada, trastornos de pánico, agorafobia, abuso del alcohol, dependencia del alcohol, abuso de drogas, dependencia de drogas (incluso nicotina), personalidad antisocial, trastorno de somatización, estrés postraumático, esquizofrenia $\mathrm{y}$ trastornos esquizomorfos. También se evaluó el deterioro psicoorgánico.

Para generar los diagnósticos se emplearon los programas computacionales CIDI 1,0 y 1,1, una vez ingresada la información en dos ocasiones e identificadas y corregidas las incongruencias lógicas (18).

\section{Entrevistadores y capacitación}

La mayor parte de los 64 entrevistadores del estudio se seleccionaron entre candidatos pertenecientes a los últimos años de carreras del área de ciencias sociales. No se incorporaron estudiantes de medicina ni médicos, para evitar eventuales sesgos en la recopilación de los datos. La capacitación se impartió con arreglo al protocolo de la OMS, que consiste en 80 horas de instrucción teórica y de sesiones prácticas. Solo 39\% de los que recibieron la capacitación fueron aceptados finalmente como entrevistadores.

En el estudio de campo, las entrevistas se grabaron cuando los entrevistados dieron su consentimiento, lo que ocurrió en una proporción de $80 \%$. Cuando se tenía, la grabación se utilizó para corregir eventuales discrepancias en los formularios de entrevista; en caso contrario, el formulario se devolvía al entrevistador para que lo completara en una segunda cita con el entrevistado. Además, los supervisores de campo visitaron al azar una muestra de viviendas, para evaluar y verificar que las entrevistas se hubie- ran realizado en forma completa y con la persona indicada.

\section{Análisis de datos}

Una vez completados el diseño muestral y la ponderación, se estimaron los errores estándar por el método de las series lineales de Taylor, con el paquete estadístico SUDAAN. ${ }^{3}$ Se aplicó en el análisis el criterio de no reemplazar a los que no respondieron. La región, provincia, comuna y distrito seleccionados se definieron como estrato. Para examinar los factores de riesgo demográficos se empleó la regresión logística, con un intervalo de confianza de $95 \%$.

\section{Consentimiento fundamentado}

El Comité de Ética de la Universidad de Concepción revisó y aprobó cada uno de los estudios. Todos los entrevistados entregaron una declaración formal por escrito en la que consentían la entrevista. Los formularios no contenían el nombre del entrevistado y toda la información se procesó anónimamente. Se dio a los entrevistados la oportunidad de obtener el resultado de sus entrevistas CIDI; una proporción de $42 \%$ pidió esta información, de modo que es posible que el procedimiento haya aumentado la motivación para participar el estudio.

\section{RESULTADOS}

\section{Uso de servicios generales y de salud mental, por todos los entrevistados}

Más de $44 \%$ de los entrevistados usaron algún tipo de servicio de salud durante los 6 meses previos al estudio. Cerca de $17,7 \%$ de la población recibió atención no especializada de salud mental en centros de atención primaria o de salud general durante el mismo

SUDAAN, versión 7,5 (1997). Research Triangle Institute. Research Triangle Park, North Carolina, Estados Unidos. período, y solo 5,6\% recibió servicios en el sector especializado del sistema de salud. Durante ese mismo lapso 3,3\% de la población pasó por lo menos una noche en el hospital por problemas de salud física o psíquica.

En general las mujeres acuden a algún servicio de salud con mayor frecuencia que los varones $(44,2 \%$ frente a $\left.39,3 \% ; \chi^{2}=6,95 ; \mathrm{gl}=1 ; P<0,02\right)$ y preferentemente en servicios no especializados $\left(17,7 \%\right.$ frente a $11,2 \% ; \chi^{2}=10,84$; $\mathrm{gl}=1 ; P<0,003)$. No se encontraron diferencias significativas por sexo en el uso de servicios especializados de salud mental.

En términos de grupos de edad, los entrevistados de 55 a 64 años son quienes presentan la mayor tasa de uso de servicios de salud general $(55,5 \%$; $\left.\chi^{2}=20,52 ; \mathrm{gl}=4 ; P<0,005\right)$ y el grupo entre 15 y 24 años tiene la tasa más alta de uso de servicios especializados de salud mental $\left(8,6 \% ; \chi^{2}=9,70 ; \mathrm{gl}=5\right.$; $P<0,006)$. En cuanto al estado civil, son los viudos los que más usan los servicios generales $\left(52,5 \% ; \chi^{2}=44,39\right.$; $\mathrm{gl}=4 ; P<0,02)$, mientras que los que mantienen una relación de convivencia utilizan con mayor frecuencia los servicios especializados $\left(95 \% ; \chi^{2}=\right.$ 31,76; $\mathrm{gl}=4 ; P<0,001)$. Los niveles educacional y de ingresos no pronostican el uso de ningún tipo de servicios.

Como era de esperar por lo reducido de la oferta (cuadro 1), los entrevistados de Cautín son los que menos utilizaron cualquier servicio de salud en el período de estudio de 6 meses $(36,1 \%$; $\left.\chi^{2}=19,26 ; \mathrm{gl}=3 ; P<0,002\right)$; de igual modo, son los que menos utilizaron servicios no especializados de salud mental. La tasa de uso de cualquier servicio de salud fue de $25,5 \%$ en Santiago, 18,5\% en Concepción, 26,1\% en lquique y $12,1 \%$ en Cautín. Las tasas de uso de servicios especializados de salud mental en las cuatro provincias fueron de $5,2,6,8,5,6$ y $3,4 \%$ respectivamente.

\section{Uso de servicios de salud mental por personas con diagnósticos positivos}

Aun cuando las personas con algún diagnóstico positivo en el período de 
CUADRO 1. Recursos públicos de salud, por 100000 habitantes, disponibles en cuatro provincias de Chile y en todo el país (2001)

\begin{tabular}{lrrrrr}
\hline \multicolumn{1}{c}{ Recursos } & Santiago & Concepción & Iquique & Cautín & Todo el país \\
\hline Camas psiquiátricas & 34,2 & 4,8 & 32,4 & 2,2 & 21,5 \\
Médicos & 801,2 & 439,3 & 151,9 & 158,6 & 547,3 \\
Médicos de atención primaria & 185,8 & 169,7 & 61,6 & 57,0 & 151,0 \\
Psiquiatras & 28,9 & 5,7 & 2,9 & 2,2 & 17,4 \\
Equipo de consultorías & 0,3 & 0,4 & 1,2 & 0,5 & 0,4 \\
Plazas en hospital de día & 4,3 & 7,6 & 6,2 & 10,5 & 5,9 \\
Plazas en hogar protegido & 7,7 & 2,7 & 12,7 & 2,0 & 5,8 \\
Plazas en club social & 6,7 & 17,1 & 86,2 & 17,4 & 10,2 \\
Plazas en centro de día & 8,0 & 11,3 & 20,5 & 16,8 & 10,7 \\
Plazas en trabajo protegido & 4,4 & 3,0 & 6,2 & 2,9 & 3,6 \\
\hline
\end{tabular}

Fuente: Informe Estadístico Anual del Ministerio de Salud de Chile.

6 meses tuvieron una tasa más alta de hospitalización $(6,4 \%)$ que las que no habían recibido ningún diagnóstico psiquiátrico $(2,4 \%)$, la diferencia no fue estadísticamente significativa $\left(\chi^{2}=\right.$ 3,54; $\mathrm{gl}=1 ; P<0,06)$. Al considerar trastornos específicos, se advierte que las personas con el diagnóstico de un trastorno del apetito se han hospitalizado - como es de esperar- en una proporción mayor $(55,3 \%)$, seguidas de aquellas con diagnóstico de psicosis no afectivas $(23,8 \%)$, trastorno de estrés postraumático $(19,8 \%)$ y cualquier trastorno de ansiedad $(9,8 \%)$.

En el cuadro 2 se reseña la utilización que hacen de los servicios los entrevistados que presentaron un diagnóstico específico durante los 6 meses estudiados.

Destaca que $99,3 \%$ de las personas con un diagnóstico de trastorno obsesivo-compulsivo han consultado al nivel primario de salud, seguidas de $80,4 \%$ de las que presentan trastornos de pánico. En el extremo opuesto se ubican las personas con problemas relacionados con el consumo de sustancias tóxicas, en particular el abuso de alcohol o drogas, y las que aparecen diagnosticadas con un trastorno antisocial de la personalidad. La mayor proporción de uso de servicios no especializados corresponde también a los que padecen trastornos obsesivo-compulsivos y de pánico (véase el cuadro 2). La frecuencia relativamente baja con que estas personas recurren a los servicios especializados parece reflejar, simple- mente, la muy limitada oferta que reciben.

Cuando se preguntó a los entrevistados a quién acudirían en busca de ayuda en el caso de un problema de salud mental, la mayoría no mencionó más opciones que el propio sistema formal de salud. Por ejemplo, hablar con un amigo lo plantearon $14,3 \%$ de los entrevistados y hablar con un miembro o representante de alguna iglesia solo por 2,7\% de las personas que participaron en el estudio. Además, apenas $1,2 \%$ de los entrevistados sugirieron la consulta de un curandero, machi o hierbatero (yerbero).

Más de la cuarta parte de los entrevistados $(26,5 \%)$ que acudieron a consulta médica en el período estudiado informaron la mención de algún problema psíquico en la entrevista. Por otro lado, el motivo de $43 \%$ por ciento de las consultas no fue la atención de problemas psíquicos.

De los que no buscaron atención médica, 28,5\% reconocieron que la necesitaban por algún problema físico $\mathrm{y}$ $12,7 \%$ afirmaron que debían hacerlo por un problema psicológico.

\section{Variables sociodemográficas y uso de servicios}

En cuanto a la relación entre el sexo y la utilización de servicios que hacen las personas con alguno de los diagnósticos estudiados, las mujeres usan con una frecuencia mayor "todos los servicios de salud mental" $\left(52,2 \% ; \chi^{2}=\right.$
11,08; $\mathrm{gl}=2 ; P<0,003)$ y con frecuencia menor los de "salud mental no especializados" $\left(48,2 \% ; \chi^{2}=8,51 ; \mathrm{gl}=2 ; P<\right.$ 0,006). En el caso de trastornos específicos, la única diferencia significativa se da a favor de los varones, en el trastorno por consumo de alcohol y drogas, en ambos niveles de atención $\left(\chi^{2}=\right.$ 9,78; $\mathrm{gl}=2 ; P<0,04$ y $\chi^{2}=10,12 ; \mathrm{gl}=2$; $P<0,003)$. Cabe destacar que en los cuadros graves con alta comorbilidad no hay diferencias en función del sexo en el uso de ningún tipo de servicio.

En cuanto a la edad, se advierten diferencias significativas solamente en la frecuencia de los trastornos afectivos. En este caso, las personas que menos consultan son las del grupo de 15 a 24 años de edad, tanto a todos los servicios de salud mental $\left(46,4 \% ; \chi^{2}=14,87 ; \mathrm{gl}=\right.$ 5; $P<0,03)$ como a los de salud mental no especializados $\left(37.6 \% ; \chi^{2}=14,26\right.$; gl = 5; $P<0,03)$; en estos últimos destaca también la escasa proporción en que consultan las personas con trastornos de ansiedad $\left(14,4 \% ; \chi^{2}=13,36 ; \mathrm{gl}=\right.$ 5; $P<0,04)$. Por último, el mismo grupo de 15 a 24 años acude en una proporción significativamente menor a los servicios especializados cuando presenta un diagnóstico de personalidad antisocial $\left(9,2 \% ; \chi^{2}=25,77 ; \mathrm{gl}=5 ; P<0,04\right)$.

Por otro lado, a un menor nivel de educación corresponde una frecuencia también menor de consulta a los centros especializados, con diferencias estadísticamente significativas, en especial en el grupo de analfabetos incluidos en la muestra del estudio $\left(0,0 \% ; \chi^{2}=10,08 ; \mathrm{gl}=2 ; P<0,03\right)$. Asimismo, destaca el mayor uso de servicios especializados por parte de los solteros con trastornos de ansiedad (28,2\%; $\left.\chi^{2}=12,15 ; \mathrm{gl}=4 ; P<0,03\right)$.

Por último, en comparación con las otras provincias estudiadas, Concepción es donde se utilizan con menor frecuencia todos los servicios ofrecidos $\left(\chi^{2}=24,85 ; \mathrm{gl}=3 ; P<0,0003\right)$, lo que con toda probabilidad se relaciona con la disponibilidad de recursos en el momento del estudio y no con la del año 2001 (véase el cuadro 1).

Los ingresos personales y familiares no pronostican el nivel de uso de los servicios cuando se analizan los casos con los distintos diagnósticos positivos. 
CUADRO 2. Utilización de servicios de salud mental en Chile, por adultos con diagnóstico del DSM-III-R ${ }^{\mathrm{a}}$ en los últimos 6 meses

\begin{tabular}{|c|c|c|c|c|c|c|c|c|}
\hline \multirow[b]{2}{*}{ Trastornos } & \multicolumn{2}{|c|}{$\begin{array}{l}\text { Nivel primario } \\
\text { de atención }\end{array}$} & \multicolumn{2}{|c|}{$\begin{array}{c}\text { Todos los servicios } \\
\text { de salud mental }\end{array}$} & \multicolumn{2}{|c|}{ No especializados } & \multicolumn{2}{|c|}{ Especializados } \\
\hline & $\%$ & $\mathrm{EE}^{\mathrm{b}}$ & $\%$ & $\mathrm{EE}^{\mathrm{b}}$ & $\%$ & $\mathrm{EE}^{\mathrm{b}}$ & $\%$ & $\mathrm{EE}^{\mathrm{b}}$ \\
\hline Episodio depresivo & 59,2 & 5,8 & 62,4 & 4,2 & 57,5 & 4,0 & 22,4 & 4,6 \\
\hline Episodio maníaco & 56,4 & 13,1 & 47,4 & 11,6 & 47,4 & 11,6 & 8,2 & 6,1 \\
\hline Distimia & 57,9 & 9,7 & 70,2 & 7,2 & 60,5 & 8,0 & 14,4 & 7,2 \\
\hline Cualquier trastorno afectivo & 57,1 & 6,9 & 61,0 & 3,9 & 55,0 & 4,3 & 21,4 & 4,1 \\
\hline Agorafobia sin pánico & 45,2 & 5,6 & 41,7 & 6,9 & 33,4 & 4,3 & 12,6 & 5,1 \\
\hline Trastornos de ansiedad generalizada & 52,3 & 12,0 & 66,0 & 9,1 & 66,0 & 9,1 & 15,3 & 4,8 \\
\hline Fobia social & 66,3 & 10,2 & 38,5 & 4,2 & 32,7 & 3,6 & 8,5 & 1,5 \\
\hline Fobia simple & 45,2 & 6,1 & 33,7 & 3,4 & 32,8 & 3,0 & 5,6 & 2,9 \\
\hline Trastorno obsesivo-compulsivo & 99,3 & 1,0 & 72,4 & 0,7 & 72,4 & 0,7 & 31,3 & 0,3 \\
\hline Trastorno de estrés postraumático & 45,0 & 15,4 & 64,8 & 11,1 & 64,2 & 11,1 & 12,8 & 8,2 \\
\hline Dependencia de drogas & 45,9 & 15,2 & 58,5 & 17,6 & 28,5 & 12,2 & 34,8 & 16,5 \\
\hline Dependencia de nicotina & 51,6 & 12,2 & 33,2 & 5,0 & 24,0 & 5,2 & 17,9 & 6,5 \\
\hline Cualquier trastorno de abuso & & & & & & & & \\
\hline de alcohol y drogas & 39,6 & 6,1 & 22,8 & 4,9 & 16,0 & 3,1 & 10,6 & 4,0 \\
\hline Cualquier trastorno de uso de sustan & & & & & & & & \\
\hline tóxicas & 44,2 & 7,1 & 25,5 & 3,6 & 18,7 & 2,4 & 12,0 & 3,2 \\
\hline \multicolumn{9}{|l|}{ Otros trastornos } \\
\hline Psicosis no afectiva & 52,0 & 14,0 & 53,7 & 17,2 & 46,6 & 16,6 & 9,5 & 6,6 \\
\hline Trastorno somatomorfo & 57,1 & 9,0 & 52,7 & 6,6 & 50,2 & 6,3 & 14,8 & 4,8 \\
\hline Trastorno del apetito & 59,7 & 30,6 & 61,2 & 30,3 & 55,3 & 31,3 & 6,0 & 6,9 \\
\hline Personalidad antisocial & 30,4 & 11,4 & 24,9 & 8,8 & 22,1 & 8,8 & 4,2 & 1,6 \\
\hline Cualquier trastorno ECPPc & 45,9 & 4,3 & 39,0 & 3,9 & 35,0 & 3,1 & 11,8 & 2,1 \\
\hline \multicolumn{9}{|l|}{$\begin{array}{l}\text { Comorbilidad ( } 3 \text { o más diagnósticos } \\
\text { simultáneos) }\end{array}$} \\
\hline
\end{tabular}

a DSM-III-R, versión III, revisada, del Diagnostic and Statistical Manual of Mental Disorders.

b EE, error estándar.

${ }^{\mathrm{c}}$ ECPP, Estudio Chileno de Prevalencia Psiquiátrica.

Notas: Cualquier trastorno de ansiedad no incluye la fobia social, la fobia simple ni el trastorno obsesivo-compulsivo. Psicosis no afectivas incluye la esquizofrenia, los trastornos esquizofreniformes, el trastorno esquizoafectivo, el trastorno delirante y las psicosis atípicas. Cualquier trastorno ECPP no incluye los trastornos del apetito, la fobia social, la fobia simple, el trastorno obsesivo-compulsivo ni la dependencia de la nicotina.

\section{Barreras directas e indirectas y grado de satisfacción}

En el cuadro 3 se resumen las razones que señalaron los entrevistados para no buscar tratamiento por problemas físicos o emocionales. Se efectuó el análisis estadístico respectivo para reconocer, entre las razones identificadas, las que se vinculaban con alguna característica demográfica.

$\mathrm{Ni}$ el sexo ni el tipo de diagnóstico ayuda a identificar alguna causa específica. La poca escolaridad se relaciona con la primera causa: "los problemas se resuelven solos".

Variables directas como la "preocupación por el costo", la "dificultad de traslado "y "no sabe adónde ir" apa- recen vinculadas a los más bajos niveles de ingreso y educación. Esta última variable se relaciona en mayor grado con los ingresos más bajos. Las personas casadas y de bajos ingresos afirmaron con mayor frecuencia que "el tratamiento no ayudará", y una mayoría de las casadas argumentan variables indirectas como "las dificultades de transporte" y en menor proporción aducen, como razón para no consultar, "lo que otros pudieran pensar". Los jóvenes afirmar preferentemente que pueden "resolver los problemas por sí mismos", y "el miedo al diagnóstico" fue más frecuente en las personas de bajos ingresos. No hubo factor pronóstico en relación con la "falta de confianza en los proveedores de salud".
Como se desprende del cuadro 4 , más de tres cuartas partes de las personas que participaron en el estudio refieren estar satisfechas o muy satisfechas con la atención recibida, tanto de los médicos generales como de los especialistas.

\section{DISCUSIÓN}

La información presentada corresponde al primer estudio latinoamericano acerca del uso de servicios de salud mental por parte de personas en quienes se diagnosticó algún trastorno psiquiátrico en una muestra representativa de la población en general. A pesar de su trascendencia y gran $\mathrm{co}^{-}$ 
CUADRO 3. Razones para no consultar entre quienes recibieron un diagnóstico específico en los seis meses anteriores

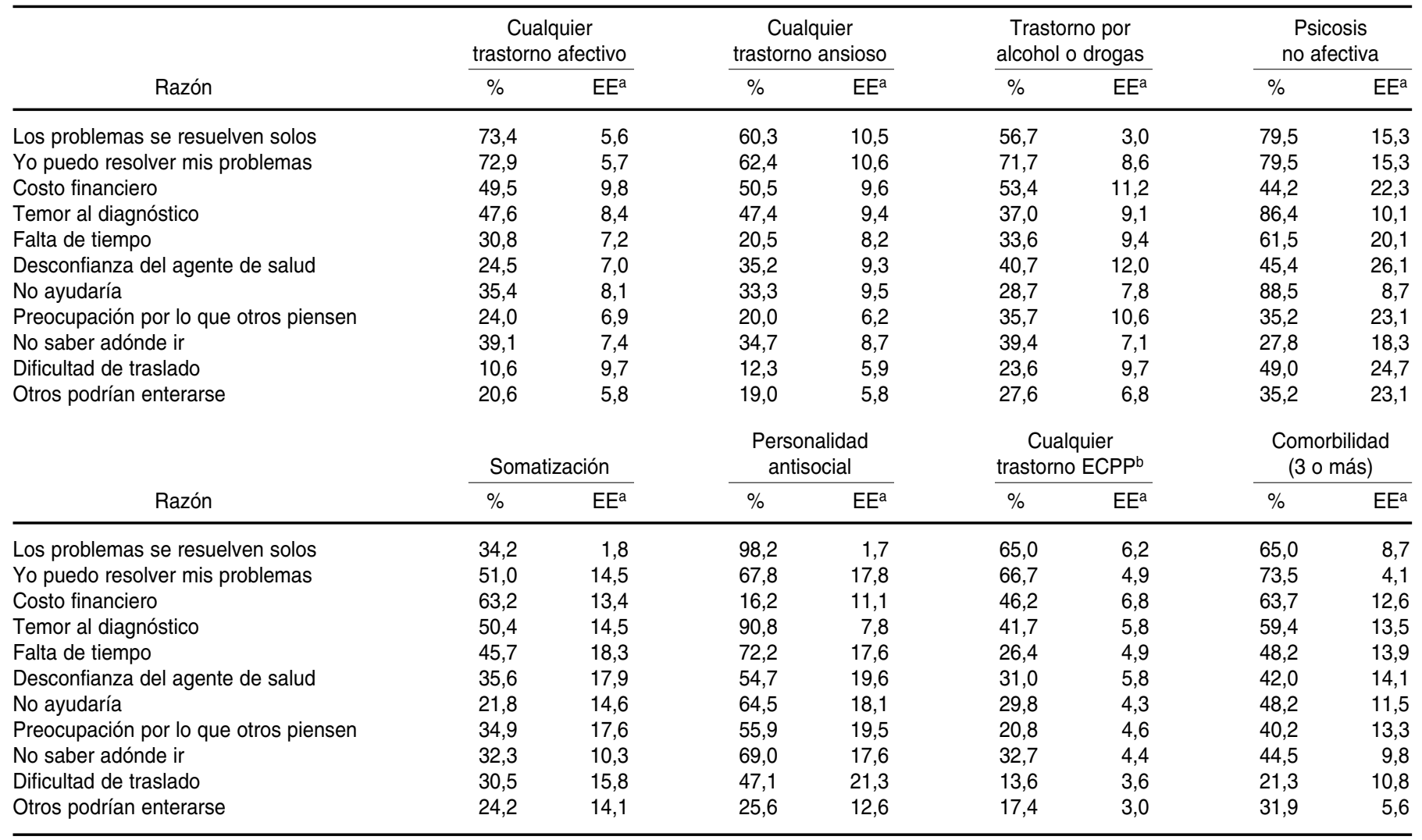

a EE, error estándar.

${ }^{\mathrm{b}}$ ECPP, Estudio Chileno de Prevalencia Psiquiátrica.

bertura, adolece de algunas limitaciones que deben considerarse al interpretar los resultados que presenta. Los diagnósticos psiquiátricos se derivaron de un instrumento de diagnóstico estructurado que aplicaron entrevistadores legos, sin confirmación clínica posterior. El lapso trascurrido entre los períodos de obtención de información en las cuatro provincias, estudiadas de forma escalonada, podría incidir, en alguna medida, en las diferencias regionales detectadas. Además, la entrevista no incluyó preguntas acerca del uso de servicios a lo largo de la vida de las personas, sino en los 6 meses previos, ni tampoco sobre eventuales intervenciones farmacológicas o psicoterapéuticas específicas.

Aun cuando la utilización global de servicios de salud mental en Chile es relativamente alta, cerca de $60 \%$ de

CUADRO 4. Grado de satisfacción con la atención ambulatoria de pacientes con algún diagnóstico del DSM-III-R en los seis meses previos al estudio

\begin{tabular}{|c|c|c|c|c|}
\hline \multirow[b]{3}{*}{ Nivel de satisfacción } & \multicolumn{4}{|c|}{ Atención } \\
\hline & \multicolumn{2}{|c|}{ Médica } & \multicolumn{2}{|c|}{ Psiquiátrica } \\
\hline & $\%$ & $\mathrm{EE}^{\mathrm{a}}$ & $\%$ & $\mathrm{EE}^{\mathrm{a}}$ \\
\hline Muy satisfecho & 22,6 & 1,6 & 22,6 & 3,0 \\
\hline Satisfecho & 56,3 & 1,7 & 54,7 & 5,6 \\
\hline Indeciso & 8,7 & 0,9 & 10,9 & 2,1 \\
\hline Insatisfecho & 8,5 & 1,3 & 10,5 & 2,7 \\
\hline Muy insatisfecho & 3,9 & 0,7 & 1,3 & 0,7 \\
\hline
\end{tabular}

los entrevistados que alcanzaron los criterios para un diagnóstico psiquiátrico no recibieron atención. Aun en el caso de diagnósticos graves, como el de psicosis no afectivas y comorbilidad, entre 20 y $45 \%$ no recibieron atención.

Tales resultados son semejantes a los obtenidos en estudios sobre el uso de los servicios en América Latina (19, 20). Aun cuando se efectuaron en poblaciones específicas, confirman la existencia de una gran brecha entre las necesidades de atención y el tratamiento efectivamente recibido.

La creencia común de que los recursos alternativos informales y folclóricos de salud se emplearían incluso masivamente y ayudarían a aminorar la brecha de atención se ve desvirtuada en Chile por los resultados del presente estudio. Los más pobres, menos instruidos y de mayor edad prácticamente no usarían recursos de ayuda informales. 
Los predictores sociodemográficos reconocidos del uso de servicios se confirmaron respecto a la mayor parte de los trastornos estudiados; destacaron en ello el sexo y el grupo de edad en riesgo de presentar la enfermedad específica, así como tener una relación de convivencia en estado de viudez.

Una proporción nada despreciable de entrevistados planteó dificultades directas para el acceso a los servicios (desinformación, costo y traslado), si bien resultan claramente mayoritarias las razones indirectas vinculadas al desconocimiento ("los problemas se resuelven solos"; "yo puedo resolver mis problemas"; "no ayudará") y el estigma ("lo que otros sepan o piensen"). No es despreciable la frecuencia con que se menciona la desconfianza en los agentes de salud, particularmente en el grupo de sujetos con trastornos relacionados con el uso de alcohol y drogas.

La poca escolaridad y la escasez de ingresos guardan estrecha relación con la identificación de barreras personales indirectas que se refieren en buena medida al escaso acceso a los servicios, sin la relación esperada en las alternativas informales mágico-religiosas.

En consonancia con los resultados de investigaciones obtenidos tanto en Estados Unidos como en Europa (21, 22), utiliza los servicios de salud mental en Chile una proporción importante de los participantes que no obtuvieron un diagnóstico positivo en la entrevista CIDI. Hasta $56 \%$ de los que usaron estos servicios no tenían un diagnóstico determinado, cifra comparativamente alta si se considera que en el estudio de comorbilidad de Estados Unidos estaban en esa situación 33,9\% de los participantes.
Es posible que, aunque necesitaran tratamiento, estas personas padecieran un trastorno subclínico que no alcanzaba a satisfacer los criterios, o bien, que aun cuando estos se hubieran cumplido en un pasado reciente, la entrevista no haya detectado el problema por efecto de un tratamiento eficaz. Cabe concluir, además, que gracias a la presencia de servicios de salud de cobertura nacional que están reorganizando y reforzando la atención en el nivel primario, han disminuido las barreras directas al acceso que encuentran las personas con cuadros leves o subclínicos, de modo que ya no buscan la atención especializada para resolver sus problemas.

En cuanto a los niveles de satisfacción, a pesar de una disminución relativa respecto del valor de casi $90 \%$ registrado en un estudio de grados de satisfacción con la atención psiquiátrica realizado hace dos décadas, los resultados parecen reflejar todavía una mirada complaciente hacia unos servicios que, si bien han mejorado, están lejos de alcanzar las normas mínimas satisfactorias si se comparan con las de los países desarrollados. Determinadas características socioculturales podrían también explicar esta benevolencia, aun cuando es posible que en buena medida refleje un reconocimiento de que la mayoría de los funcionarios de los diversos servicios de salud entregan su mejor esfuerzo, frente a una disponibilidad siempre limitada - a veces en grado crítico- de recursos materiales.

Al observar el "recorrido esperado" que siguen los pacientes por la red de servicios, resulta que este se cumple si los diagnósticos se consideran en su totalidad, pero se pierde si se analizan por separado. Los pacientes con cuadros más graves no acceden mayoritariamente a los servicios especializados, mientras que acuden a ellos en mayor proporción -aunque no la óptimaaquellos cuyos problemas han sido tema específico de políticas gubernamentales, como los cuadros de dependencia de diversas sustancias $y$, en cierta medida, los trastornos afectivos.

Por último, para que los países en desarrollo, como Chile, logren reducir la discapacidad que implican las enfermedades mentales, deben poner mayor énfasis en sus esfuerzos por disminuir la persistencia tanto de las barreras directas como de las indirectas.

La OMS (23) ha propuesto 10 recomendaciones cuyo cumplimiento ayudaría a reducir las brechas en el acceso a los servicios de salud mental: 1) lograr que la atención de trastornos mentales sea accesible en el nivel primario de atención; 2) dar acceso a los psicofármacos; 3) trasladar el énfasis de la atención de la institución a la comunidad; 4) educar a la población; 5) atraer la participación de la familia, la comunidad y los consumidores; 6) establecer programas nacionales de salud mental; 7) aumentar y mejorar la capacitación de los profesionales de esta área; 8) mejorar la interacción entre instituciones gubernamentales y no gubernamentales; 9) disponer de un sistema de monitoreo de servicios de salud mental que incluya indicadores de calidad; y 10) atraer aportes adicionales para apoyar la investigación en el sector. Una proporción importante de esas propuestas es todavía un desafío que debe enfrentarse en países con problemáticas similares a la de Chile.

\section{REFERENCIAS}

1. Vicente B, Vielma M, Lliapas I. Tipo y calidad de atención psiquiátrica: la opinión y niveles de satisfacción de los usuarios como una forma de evaluación. Rev Psiquiatría (Chile). 1990;7(3):509-16.

2. Vicente B, Vielma M, Jenner FA, Mezzina R, Lliapas I. User's satisfaction with mental health services. Int J Soc Psychiatry. 1993; 39(2):121-30.
3. Kessler Rc, Frank Rg, Edlund M, Katz Sj, Lin $\mathrm{E}$, Leaf P. Differences in the use of psychiatric outpatient services between the United States and Ontario. N Engl J Med. 1997;336(8):551-7.

4. Katz SJ, Kessler RC, Frank RG, Leaf P, Lin E, Edlund M. The use of outpatient mental health services in the United States and Ontario: the impact of mental morbidity and per- ceived need for care. Am J Public Health. 1997;87(7):1136-43

5. Mccreadie R, Leese M, Tilak-singh D, Loftus L, Macewan T, Thornicroft G. Nithsdale, Nunhead and Norwood: similarities and differences in prevalence of schizophrenia and utilisation of services in rural and urban areas. Br J Psychiatry. 1997;170:31-6. 
6. Vega W, Kolody B, Aguilar-Gaxiola S, Catalano R. Gaps in service utilization by Mexican Americans with mental health problems. Am J Psychiatry. 1999;156(6):928-34.

7. Katz S, Kessler R, Frank R, Leaf P, Lin E. Mental health care use, morbidity, and socioeconomic status in the United States and Ontario. Inquiry. 1997;34(1):38-49.

8. Solis LR, Medina-Mora ME. La utilización de los servicios de atención para la salud mental por mujeres mexicanas. Resultados de dos encuestas nacionales. Salud Mental (México). 1994;17(1):7-11.

9. Vicente B, Vielma M, Rioseco P. Un programa de epidemiología psiquiátrica para Chile. Rev Psiquiatría (Chile). 1994;11(4):160-7.

10. Saldivia S, Vicente B, Kohn R, Rioseco P, Torres $S$. Use of mental health services in Chile. Psychiatric Serv. 2004;1(55):71-6.

11. Vicente B, Kohn R, Rioseco P, Saldivia S, Baker CL, Torres S. 6-month and 1-month prevalence in the Chile Psychiatric Prevalence Study. Br J Psychiatry. 2004;184:299-305.

12. Kish L. Survey Sampling. New York: John Wiley \& Sons; 1965.

13. American Psychiatric Association. Diagnostic and Statistical Manual of Mental Disorders, DSM-III-R. Tercera edición, revisada. Wash- ington, D.C.: American Psychiatric Association; 1987.

14. Robins LN, Wing J, Wittchen Hu, Helzer JE, Babor TF, Burke J, et al. The Composite Internatinal Diagnostic Interview: an epidemiologic instrument suitable for use in conjunction with different diagnostic systems and in different cultures. Arch Gen Psychiatry. 1988; 45:1069-77.

15. Robins LN, Helzer JE, Croughan J, Ratcliff KS. National Institute of Mental Health diagnostic interview schedule: its history, characteristics, and validity. Arch Gen Psychiatry. 1981;38: 381-9.

16. Vielma M, Vicente B, Rioseco P, Catro P, Castro $\mathrm{N}$, Torres S. Validación en Chile de la entrevista diagnóstica estandarizada para estudios epidemiológicos CIDI. Revista de Psiquiatría. 1992;9:1039-49.

17. Rioseco P, Vicente B, Uribe M, Vielma $M$ Castro N, Torres S. El DIS-III-R: una validación en Chile. Revista de Psiquiatría. 1992;9: 1034-8.

18. Pfister H, Wittchen HU. CIDI Core Computer Manual for Data Entry and Diagnostic Programmes for the Composite International Diagnostic Interview (CIDI). Geneva: World Health Organization;1990.
19. Carveo-Anduaga JJ, Martínez NA, Rivera BE, Polo A. Prevalencia en la vida de episodios depresivos y utilización de servicios especializados [Lifetime prevalence of depressive episodes and specialized service utilization] Salud Mental (México). 1997;20(2):15-23.

20. Saldivia S. Vicente B, Kohn R, Rioseco P, Torres $S$. Use of mental services in Chile. Psychiatric Serv. 2004;55:1;71-6.

21. Kessler RC, Zhao S, Katz SJ, Kouzis AC, Frank RG, Edlund M, et al. Past-year use of outpatient services for psychiatric problems in the National Comorbidity Survey. Am J Psychiatry. 1999; 56:115-23.

22. Biji R, Graaf R, Hiripi E, Kessler R, Konh R, Offord D, et al. The prevalence of treated and untreated mental disorders in five countries. Health Aff. 2003;3(22):122-33.

23. Andrade LH, Walters EE, Gentil V, Laurenti R. Prevalence of ICD-10 mental disorders in a catchment area in the city of São Paulo, Brazil. Soc Psychiatry Psychiatr Epidemiol. 2002; 37:316-25.

Manuscrito recibido el 27 de septiembre de 2004. Aceptado para publicación, tras revisión, el 22 de abril de 2005.

ABSTRACT Objective. To describe the patterns in the use of general health services and specialized health services among adults with mental health problems in Chile, as well as those persons' level of satisfaction with the services. The overall objective was to op-

\section{Service use patterns among adults with mental health problems in Chile} timize the use of the limited resources available for mental health care in the countries of the Americas, especially Chile.

Methods. The diagnoses and the patterns of use of mental health services were obtained from the Chilean Study of Psychiatric Prevalence (Estudio Chileno de Prevalencia Psiquiatrica). That representative research on the adult population of Chile was based on a stratified random sample of 2987 people 15 years old and older, done over the period of 1992 to 1999 . The psychiatric diagnoses were obtained using the Composite International Diagnostic Interview (CIDI). The interviewees were also asked about their use of general health care services and of mental health services in the preceding six months, as well as any barriers to accessing the services.

Results. More than $44 \%$ of the interviewees had had contact with some type of health service during the six months prior to the study, but only $5.6 \%$ received specialized care. Those who presented with a diagnosis of obsessive-compulsive disorder or of panic disorder consulted more frequently, but not in specialized centers. Consumption of substances such as alcohol and drugs as well as antisocial personality disorder were associated with a low level of consultation. When asked about sources of assistance for mental health problems, the majority of the interviewees mentioned only the formal health care system. More than $75 \%$ of the interviewees said that they were satisfied or very satisfied with the care that they had received.

Conclusions. Our results confirm the existence of a wide gap between the need for care and the treatment that is actually received. The informal and folkloric alternative resources (priests, family members, healers, herbalists, etc.) were used less frequently than is generally believed to be true. The indirect barriers of access to services-linked to a lack of knowledge and to stigma-were more frequent than were the direct barriers. A sizable number of people who did not have a positive diagnosis on the CIDI utilized mental health services.

Keywords Community mental health services, mental disorders, patient satisfaction, Chile. 